\title{
Traumatic Posterior Fossa Extradural Haematoma
}

\author{
Roka YB, ${ }^{1}$ Kumar P, ${ }^{1}$ Bista P, ${ }^{1}$ Sharma GR, ${ }^{1}$ Adhikari D' \\ 'National Neurosurgical Referral Centre, National Academy of Medical Sciences Kathmandu, Nepal
}

\begin{abstract}
Posterior fossa extradural haematoma is known for the vague signs and symptoms and a notorious course that varies from recovery to sudden death. The incidence of posterior fossa epidural hematomas among intracranial epidural hematomas has been reported from $4 \%$ to $7 \%$. Subsequently, PFEDH with low GCS or the haematoma of more than $10 \mathrm{ml}$ were subjected to evacuation. Since the volume of the posterior fossa is limited, patients deteriorate early with the development of obstructive hydrocephalus, which is visible in the CT scan in only thirty percent of cases.
\end{abstract}

A retrospective study of 43 cases was done in this Institute from May 1999 to December 2005. The males $(98 \%)$ have a clear predominance over female patients $(2 \%)$. Road traffic accidents accounted for the majority of the cases $(80 \%)$, fall for the rest $(17 \%)$ and one case due to a bullhorn injury. Vomiting was the most common symptom accounting for $67 \%$ of cases followed by transient loss of consciousness in $48 \%$ and headache in 34\%. On arrival to the hospital $67 \%$ presented with a GCS more than $13,28 \%$ with score of $9-12$ and the rest $5 \%$ with GCS of less than 8 . Out of the total 43 cases of PFEDH surgical evacuation was done in 33(76\%) and conservative management in 10 cases (23\%). A dichotomised Glasgow outcome score was used to measure the outcome. This was favorable in 27 of the 33 cases operated $(81 \%)$, and 7 out of the 10 conservatively managed group $(70 \%)$. Overall favorable outcome was found in 34 cases $(79 \%)$ with overall mortality of the study being $7 \%$.

Key words: extradural, haematoma, posterior fossa, trauma

\section{INTRODUCTION}

Posterior fossa extradural haematoma (PFEDH) is known for the vague signs and symptoms and a notorious course that varies from recovery to sudden death. The incidence of posterior fossa epidural haematomas among intracranial epidural haematomas has been reported to be $4 \%$ to $7 \%$. Since the volume of the posterior fossa is limited, patients deteriorate early with the development of obstructive hydrocephalus, which is visible in the computed tomography (CT) scan in only thirty percent of cases. The most frequent symptoms are headache, stiff neck, vomiting and a deteriorating level of consciousness. A classification of

\footnotetext{
Correspondence:

Dr. Yam Bahadur Roka

National Neurosurgical Referral Centre

National Academy of Medical Sciences

Bir Hospital, Kathmandu, Nepal

Phone: +977-9841256560

dryamroka@yahoo.com
} 
Roka et al. Traumatic Posterior Fossa Extradural Haematoma

extradural haematoma based on the interval between the traumatic event and operation is well recognized. They may be acute onset (that is, less than 24 hours), subacute (between 24 hours to 10 days) and chronic (that is, after 10 days). Subsequently, PFEDH with low Glasgow Coma Score (GCS) or the haematoma of more than $10 \mathrm{ml}$ were subjected to evacuation.

\section{MATERIAL AND METHODS}

This is a retrospective study of 43 patients of posterior fossa extradural haematoma (PFEDH), out of a total 432 cases of Extradural Haematomas. The study period is from May 1999 to December 2005 and conducted at National Neurosurgical Referral Centre, Bir Hospital, Kathmandu, Nepal. Ethical approval was taken from the institute ethical committee. Data was calculated using Microsoft office excel 2007.

\section{RESULTS}

The total number of PFEDH cases was forty three out of a total of four hundred and thirty two cases of EDH. This gave an incidence of $10 \%$. The most common age was in the 21-30 year group (16 cases) followed by $11-20$ year (13). There were 5 cases less than 10 years and 2 cases more than 40 years. This showed predominance of these haematoma in the productive age group. The age ranged from 5 to 62 years (Table 1).

The males (98\%) have a clear predominance over female patients $(2 \%)$. Road traffic accidents accounted for the majority of the cases $(80 \%)$, fall for the rest $(17 \%)$ and one case due to a bullhorn injury. The most common mode of fall was from the tree and in the toilet (Table 2).

Vomiting was the most common symptom accounting for $67 \%$ of cases followed by transient loss of consciousness in $48 \%$, headache in $34 \%$ and incontinence of urine $(2 \%)$ and cranial nerve palsies $(4 \%)$ in the rest. In the majority multiple episodes of vomiting was the main complaint at presentation ( $80 \%$ ) while the rest developed it later. One case of 12 year old boy presented to us 7 days later with only headache. LOC was present with a range of few minutes to state of unconsciousness at admission in $5 \%$. The cause for a case of VII cranial nerve palsy was a fracture of the petrous bone while the other case of VI CN palsy was uncertain (Table 3). On arrival to the hospital $67 \%$ presented with a GCS more than $13,28 \%$ with score of $9-12$ and the rest $5 \%$ with GCS of less than 8 . Majority of the patients presented within 6 hours of the incident although it varied to 7 days in two cases, this shows the important observation that most of these cases have trivial symptoms and findings. On plain $\mathrm{X}$-ray investigation $53 \%$ had occipital fracture and $13 \%$ had associated cervical fracture(Table 4).
CT scan showed location of the haematoma on right $(81 \%)$ and the rest in the left. The probable explanation may be the presence of a larger transverse sinus on the right side which is more prone to traumatic bleed. Pure PFEDH was found in 27 cases (62\%) and it was mixed in 16 cases $(37 \%)$. In the mixed category there were two cases of bilateral supra and infra tentorial EDH $(12 \%)$. Supratentorial contusion was present in 8 cases $(18 \%)$ mainly in the frontal and parietal regions. There was accompanying hydrocephalus in $20 \%$ of cases. Hydrocephalus was mild with blowing of the temporal horns on the CT scan. None of the hydrocephalus patients needed VP shunt in the pre and post operative period (Table 5).

The contusion was located in frontal, parietal and temporal areas. Haematoma volume was calculated in all of the cases and more than $10 \mathrm{ml}$ found in 33 of the cases $(76 \%)$. The calculation was done as per the formula of $\mathrm{L}$ (AP diameter) $\times \mathrm{B} \times \mathrm{H}$ divided by 2 to give the volume in $\mathrm{ml}$.

A protocol of surgery if the volume was more than 10 $\mathrm{ml}$, deteriorating GCS and features of mass effect on CT scan was strictly adhered to and surgery done for cases that qualified for this. Out of the total 43 cases of PFEDH surgical evacuation was done in $33(76 \%)$ and conservative management in 10 cases $(23 \%)$. Surgery consisted of doing occipital craniectomy and evacuation of the haematoma (Figure 1). The most common cause for the haematoma was bleeding from the transverse sinus followed by oozing from a occipital bone fracture. All cases with supratentorial haematomas were managed with post operative ICP monitoring for 48 hours to predict possible increase in the haematoma. None of them needed further surgery for the supratentorial haematomas. In two cases there was bilateral supra and infra tentorial haematoma and we did a supratentorial craniotomy with suboccipital craniectomy bilaterally. Out of the total ten cases that were managed conservatively three of them deteriorated suddenly and expired. One case of PFEDH associated with frontal contusion showed increase in size of the latter on repeat scan and needed evacuation although the PFEDH remained the same. This patient had a fall in GCS that mandated a repeat scan. Rest of the seven cases did not need surgical intervention.

All patients were followed up as per the unit protocol at the end of 2 weeks, 6 weeks and three months and GOS scale was used at the end of three months. A dichotomised Glasgow outcome score was used to measure the outcome. This was favorable in 27 of the 33 cases operated ( $81 \%)$, and 7 out of the 10 conservatively managed group (70\%). The overall favorable outcome was found in 34 cases $(79 \%)$. The mortality, which was in the nonoperative group, 
Roka et al. Traumatic Posterior Fossa Extradural Haematoma

was $10 \%$, overall mortality of the study was $7 \%$. The unfavorable group consisted with patients with GCS less than 9 (Table 6).When compared to the initial admission GCS there was recovery in 4 patients (33\%) out of 12 in the GCS less than nine group.

This would suggest that in all symptomatic patients surgery is the treatment of choice. We have since then advocated active surgery in all symptomatic patients that fit into our criteria for surgery. These are, low GCS on admission, volume more than $10 \mathrm{ml}$, deteriorating GCS and presence of hydrocephalus.

\section{DISCUSSION}

Extradural posttraumatic posterior fossa hematoma is a rare condition estimated to complicate about $0.3 \%$ of all craniocerebral injuries, and represents $4 \%$ to $12.9 \%$ of the entire group of extradural hematomas. ${ }^{1}$ Due to the small volume of the posterior fossa and contained important structures mortality can be high if the haematoma is missed. A high index of suspicion is needed for timely intervention to prevent death. We had three mortalities during our early study period where we were less aggressive and since then our indication has changed based on our protocol. Since then there has been no deaths.

PFEDH accounts for even fewer incidences in children $(1.3 \%)$. Sudden deterioration is a feature that distinguishes it from the supratentorial extradural haematomas. ${ }^{2}$ Developing hydrocephalus and the presence of vague clinical signs often mislead into delay in surgical intervention. Hydrocephalus may be observed in as high as 30 percent of cases on the CT scan. Presence of occipital haematoma, swelling, site of impact and often Battles sign are clue to its diagnosis. Fracture of the occipital bone is and important sign that mandates observation and repeat san later to diagnose these haematomas. Change in GCS or severe headache with vomiting and new onset cerebellar signs are associated features that help to clinch the diagnosis. Although isolated vomiting has no relevance in head injury we find that when it comes to PFEDH it is the main symptom and it cannot be ignored if we are suspecting these haematoma. Some studies have shown that neck stiffness and drowsiness were the commonest clinical signs. ${ }^{3} \mathrm{CT}$ scan remains the choice of investigation to detect these haematomas. The presence of haematoma more than $10 \mathrm{ml}$, hydrocephalus and displacement of the IV ventricle are all indications for surgery. These haematomas may be confused with cerebellar contusion and $\mathrm{SAH}$. The presence of dura between the cerebellar cortex and the haematomas help to separate these haematomas. There have been various classification systems proposed based upon the obliteration of perimesencephalic cisterns and/or displacement of the fourth ventricle or midline shift more than $5 \mathrm{~mm}$, volume more than $10 \mathrm{ml}$, a thickness of more than $15 \mathrm{~mm}$ and the absence of a significant intracranial haematoma elsewhere. ${ }^{4,5}$

Surgery remains the gold standard for the treatment of symptomatic PFEDH. This may be in the form of suboccipital craniectomy or craniotomy or both depending on the size of the haematoma. A case of Bilateral posterior fossa EDH with supratentorial extension was operated by bilateral suboccipital craniectomy and supratentorial occipital craniotomy with good outcome. ${ }^{10}$ The PFEDH may be associated with coexisting supratentorial lesions. In the literature, supratentorial pathologies associated with PFEDH have been reported in as high as 50\%-87.5\%. ${ }^{6}$ This may be in the form of haematoma or contusion. A contre coup mechanism may be the explanation for such injuries. A decision needs to be made as to which is more life threatening and surgery undertaken. We have operated in these lesions with no increase in the PFEDH and conversely the latter may be removed leaving the supratentorial lesion. Although it may seem to affect the overall prognosis but then some studies have proven that these coexisting intracranial lesions may have no influence on mortality. ${ }^{7}$ Conservative management is another option if the patient is symptomless and he is under close monitoring in the neurosurgical ICU. There have also been case reports of these haematomas resolving spontaneously without any intervention. ${ }^{8}$

The rapidity of onset and initial GCS are the main factors that determine the overall prognosis. Acute haematomas often carry a high mortality risk with the range of $12 \%-70 \%$. Haematomas that present late are usually venous in origin and there are many reports of such chronic PFEDH in literature.

We have compared our results with other studies (table 7). 4,9 When compared with the Pure PFEDH our study has a higher number of these cases. In terms of GCS at presentation the majority are more than score of 8 in all the groups whereas both ours and Edson et al have shown that more than two thirds are having GCS more than 14. The striking feature is that we have been less aggressive than the others in terms of surgical management. Only $76 \%$ of our cases underwent surgery compared with $93 \%$ of the rest. In spite of the high rate of conservative management our mortality rates are more favourable than the others.

We took these as the number of cases match with each other. We find that the rate of surgical intervention is high in these studies and majority present with good GCS. The haematomas also seem to be pure in most of the cases. All these studies indicate that aggressive surgical management is a must in all cases of PFEDH to prevent morbidity and mortality. 
Roka et al. Traumatic Posterior Fossa Extradural Haematoma

Table 1. Age distribution

\begin{tabular}{ll}
\hline Age & No (\%) \\
\hline$<10$ & $5(11.6)$ \\
$11-20$ & $13(30)$ \\
$21-30$ & $16(37)$ \\
$31-40$ & $7(16)$ \\
$>40$ & $2(4.6)$ \\
Total & 43 \\
\hline
\end{tabular}

Table 2. Mode of presentation

\begin{tabular}{ll}
\hline Mode & No $(\%)$ \\
\hline RTA & $33(76.7)$ \\
Fall & $9(20.9)$ \\
Animal assault & $1(2.3)$ \\
Total & 43 \\
\hline
\end{tabular}

Table 3. Clinical Presentation:

\begin{tabular}{ll}
\hline Vomiting & $29(67.4 \%)$ \\
Transient LOC & $21(48.8 \%)$ \\
Headache & $15(34.8 \%)$ \\
Incontinence & $1(2.3 \%)$ \\
VI CN palsy & $1(2.3 \%)$ \\
VII CN palsy & $1(2.3 \%)$ \\
\hline
\end{tabular}

Table 4. State of Consciousness

\begin{tabular}{ll}
\hline GCS & No.(\%) \\
\hline $13-15$ & $29(67 \%)$ \\
$9-12$ & $12(25 \%)$ \\
$<9$ & $2(5 \%)$ \\
\hline
\end{tabular}

Table 5. CT Scan findings

\begin{tabular}{ll}
\hline Location & \\
\hline Right & $35(81 \%)$ \\
Left & $8(19 \%)$ \\
Type & $16(37 \%)$ \\
Mixed (Supra \& Infratentorial) & $27(62 \%)$ \\
Pure (Infratentorial) & $8(18 \%)$ \\
Accompanying Features & $9(20 \%)$ \\
Supratentorial contusion & \\
Hydrocephalus & $33(76 \%)$ \\
Volume & \\
More than $10 \mathrm{ml}$ &
\end{tabular}

Table 6. Glasgow Outcome Score (GOS) outcome at three months

\begin{tabular}{ll}
\hline GOS & Percentage \\
\hline Favourable & $33(79 \%)$ \\
Unfavourable & $7(16 \%)$ \\
Expired & $3(10 \%)$ \\
\hline
\end{tabular}

Table 7. Comparison of results

\begin{tabular}{llll}
\hline Study & $\begin{array}{l}\text { Pozzati et } \\
\text { al, }^{1989} \mathbf{9}^{9}\end{array}$ & $\begin{array}{l}\text { Edson et } \\
\text { al,2004 }\end{array}$ & Our study \\
\hline Total Patients & 32 & 43 & 43 \\
Pure PFEDH & 16 & 21 & 27 \\
GCS $>14$ & 11 & 31 & 29 \\
GCS $8-13$ & 11 & 7 & 12 \\
GCS $<7$ & 10 & 5 & 2 \\
Surgery & $30(93 \%)$ & $40(93 \%)$ & $33(76 \%)$ \\
Mortality & $15 \%$ & $20 \%$ & $7 \%$ \\
\hline
\end{tabular}
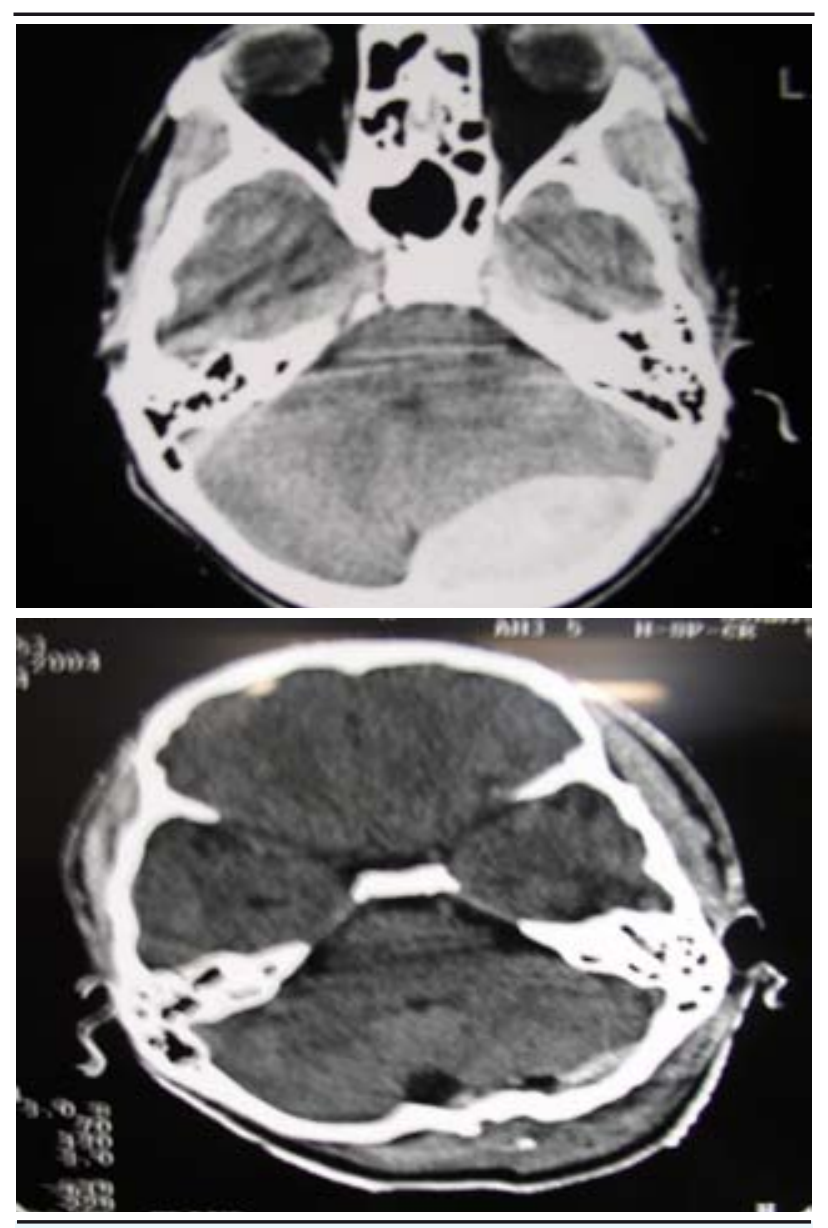

Figure 1. Pre and post operative CT findings

\section{CONCLUSIONS}

The posterior fossa is an uncommon site for epidural haematomas. Clinical progress is silent and slow, but the deterioration is sudden and quick to become fatal if not promptly treated. Early recognition is therefore extremely important. The recommended treatment for posterior fossa epidural hematoma is surgical evacuation soon after the diagnosis, since the posterior fossa contains vital structures. PFEDH is common in the younger generation and this warrants careful observation and timely surgical intervention to prevent 
Roka et al. Traumatic Posterior Fossa Extradural Haematoma

rapid and fatal deterioration. The haematoma of more than $10 \mathrm{ml}$ size should be evacuated even if the GCS suggests mild head injury. A prospective controlled trial study is required to find the critical volume of the PFEDH to be subjected for the surgery.

\section{REFERENCES}

1. Garza-Mercado R. Extradural hematoma of the posterior cranial fossa. Report of seven cases with survival. Journal of Neurosurgery 1983 Oct; 59(4):664-72.

2. Costa Clara JM, Claramunt E, Ley L, Lafuente J. Traumatic extradural hematomas of the posterior fossa in children. Childs Nerv Syst 1996 Mar; 12(3):145-8.

3. Mahajan RK, Sharma BS, Khosla VK, et al. Posterior fossa extradural haematoma-experience of nineteen cases. Ann Acad Med Singapore 1993 May; 22(3 Suppl ):410-3.

4. Bozbuga M, Izgi N, Polat G, Gurel I. Posterior fossa epidural hematomas: observations on a series of 73 cases. Neurosurg Rev 1999; 22(1):34-40.

5. Wong CW. The CT criteria for conservative treatment--but under close clinical observation-of posterior fossa epidural haematomas Acta Neurochir (Wien) 1994; 126(2-4):124-7.
6. Zuccarello M, Pardatscher K, Andrioli GC, Fiore DL, Ýavicoli R, Cervellini P. Epidural hematomas of the posterior cranial fossa. Neurosurgery 1981; 8:434-437.

7. Koc RK, Pasaoglu A, Menku A, Oktem S, Meral M. Extradural hematoma of the posterior cranial fossa. Neurosurg Rev 1998; 21(1):52-7.

8. Kang SH, Chung YG, Lee HK.Rapid disappearance of acute posterior fossa epidural hematoma. Neurol Med Chir (Tokyo) 2005 Sep; 45(9):462-3.

9. Pozzati E, Tognetti F, Cavallo M, Acciarri N. Extradural hematomas of the posterior cranial fossa. Observations on a series of 32 consecutive cases treated after the introduction of computed tomography scanning. Surg Neurol 1989 Oct; 32(4):300-3.

10. Devkota UP, Kumar P, Roka YB. Surgery for posterior fossa extradural haematoma: Case report. PMJN 6;1:2006. 